\title{
The Magellanic Catalogue of Stars (MACS) ${ }^{\star}$
}

\author{
H.-J. Tucholke ${ }^{1,2}$, K.S. de Boer ${ }^{2}$ and W.C. Seitter ${ }^{1}$ \\ 1 Astronomisches Institut der Universität Münster, Wilhelm-Klemm-Straße 10, D-48149 Münster, Germany \\ 2 Sternwarte der Universität Bonn, Auf dem Hügel 71, D-53121 Bonn, Germany
}

Received November 23, 1995; accepted February 2, 1996

\begin{abstract}
We have compiled the Magellanic Catalogue of Stars (MACS), a catalogue of positions for 243561 stars covering large areas around the Large and Small Magellanic Cloud (LMC and SMC). The positions have been measured using ESO Schmidt plates and refer to the FK5 system via the PPM catalogue. A comparison of positions from different plates shows internal errors at a level of $0{ }^{\prime \prime} 15$ to $0{ }^{\prime \prime} 23$. The positional accuracy is estimated to be better than 0.5 per coordinate for $99 \%$ of the stars. The limiting magnitude of the MACS is $B \leq 16$. 3 , but the catalogue is not complete: only those stars are included which are undisturbed by close neighbours as verified by visual screening in order to obtain a clean astrometric reference. We introduce for the MACS a nomenclature and numbering system in accord with IAU rules based on position, leaving the possibility to add further stars without upsetting the numbering system. The catalogue is available via the Centre des Données Strasbourg (CDS).
\end{abstract}

Key words: Magellanic Clouds — catalogs — astrometry — galaxies: stellar content

\section{Introduction}

The Magellanic Clouds harbour a vast number of objects of interest for the observer. In many cases it is, however, difficult to find accurate coordinates for stars in crowded fields of the Clouds. These are needed for telescope pointing and for comparison with observations in other wavelengths. For example, the IUE satellite needs input positions better than 1 .' 5 for proper observation, while the accuracy requirements for fiber positioning in multi-object spectroscopy are more demanding.

A first step to solve this problem was made by Périé et al. (1991), who published a catalogue of almost 1000 stars in the direction of the Magellanic Clouds with typical $V$ magnitudes between 9 and 11 . The positions are based on ESO Schmidt plates and have a positional accuracy of 0 .'4. This catalogue can be used as reference catalogue to obtain star positions on, e.g., astrographic plates.

In order to have enough reference stars for CCD's, however, a much higher surface density of stars is needed. The Guide Star Catalogue (GSC) Version 1.0 (Lasker et al. 1990) reaches $V$ magnitudes of about $15^{\mathrm{m}}$, but probably contains many individual errors due to the severe crowding conditions in the Magellanic Clouds. In addition, the positions contain field-dependent systematic errors which

Send offprint requests to: H.-J. Tucholke (email tucholke@astro.uni-bonn.de)

${ }^{\star}$ Based on observations at the European Southern Observatory, La Silla, Chile may reach 1".5 (Taff etal. 1990a). Comparison of GSC 1.0 positions in overlap regions (where the positions derived from different plates are given individually) show that Magellanic Cloud star positions may have individual errors even larger than this. Another deep catalogue is the COSMOS/UKST Southern Sky Object Catalogue (Yentis et al. 1992), whose content and astrometric accuracy is described by Drinkwater et al. (1995). It reaches $B_{\mathrm{J}} \leq 21.5$; due to crowding problems, however, the regions of the Magellanic Clouds have been left out for the time being.

These examples show that it is desirable to pay attention to the possible influence of crowding on the accuracy of individual star positions and to control the systematic errors in position which are introduced by Schmidt telescopes. De Boer (1993) presented plans for a catalogue with these features. The present paper describes the construction of its first version. The Magellanic Catalogue of Stars (MACS) is based on scans of ESO Schmidt plates and contains about 244000 stars covering large areas around the LMC and the SMC. The limiting magnitude is $B \leq 16^{\mathrm{m}} 3$. The stars of this catalogue have been screened interactively to ascertain that they are undisturbed by close neighbours.

The present version of the MACS contains neither proper motions nor colours. In addition, in very crowded regions the identification with other observations is difficult, since many disturbed stars have been excluded. All 
these shortcomings should be alleviated by future extensions of the catalogue.

\section{Observations and object selection}

\subsection{Plate material}

The MACS is based on plates taken with the ESO Schmidt telescope in 1988/1989. A few plates taken 1991 were also used. The exposure time is 60 minutes in the blue passband IIa-O + GG 385. The limiting magnitude is $B \approx$ $20 \mathrm{~m} 5$. The plate centres are the same as in the ESO/SERC Survey. The plates were taken with parameters as similar as possible to the ESO Quick Blue Survey (West \& Schuster 1982). The original aim was the derivation of absolute proper motions of the Magellanic Clouds with respect to background galaxies (Tucholke \& Hiesgen 1991). We used 21 plates of 12 fields, so that most fields were covered by two plates. The MACS in its present version covers areas of about 200 and 120 square degrees for the LMC and the SMC, respectively (Figs. 1 and 2). Table 1 gives the epochs and J2000.0 centres for the plates used.

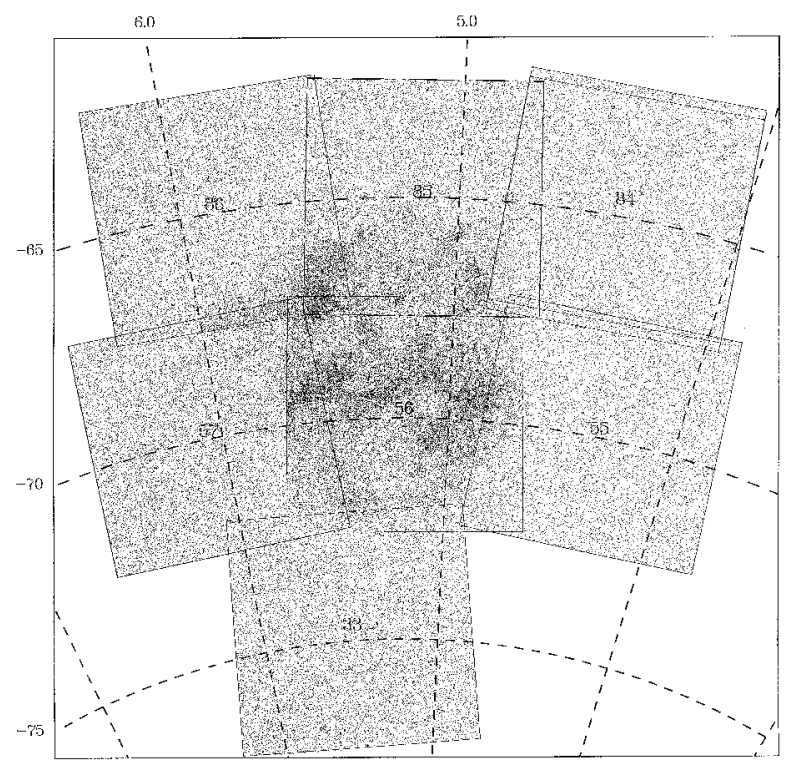

Fig. 1. Sky distribution of 175779 catalogue stars towards the LMC. Also given are numbers identifying the ESO/SERC atlas fields, the outlines of the plates used, and a J2000.0 coordinate grid. The largest concentrations of catalogue stars are found near active star-forming regions, while the bar contributes relatively few stars brighter than $B \approx 16.3$

\subsection{Measurement and object detection}

All plates were digitized with the PDS $2020 \mathrm{GM}^{\text {plus }}$ microdensitometers of the Astronomical Institute of Münster University, using a step size of $15 \mu \mathrm{m}$ or $1^{\prime \prime}$. A full plate scan has $19000 \times 19000$ pixels. The positional

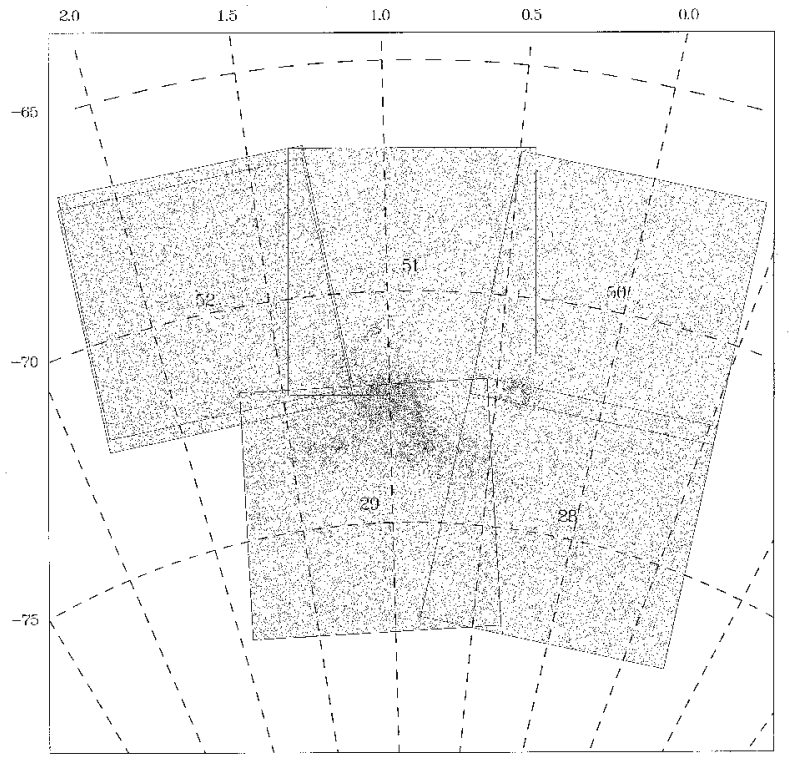

Fig. 2. Sky distribution showing 67782 catalogue stars in the direction of the SMC as in Fig. 1. The outer wing in the SE is not yet included in the catalogue. Note the concentrations around the galactic globular clusters 47 Tuc and NGC 362

drift of the measuring machines was controlled by scanning 7-9 randomly chosen stars before and after the large scan. Typical drifts were 1 to $2 \mu \mathrm{m}$.

A fully automatic program (Horstmann 1992) was used for the detection of objects. For the MACS, the parameters of this program were chosen to yield a limiting magnitude of about $B=16^{\mathrm{m}} \cdot 3-16^{\mathrm{m}} 5$, which was selected to yield a sufficient surface density of catalogue stars. The magnitude limit was checked using various photoelectric and CCD photometry measurements from the literature. Since published photometry is not available for all fields, we had to rely on the homogeneity of the plate material. From comparison of the internal magnitudes in plate overlap regions (see below) we found that the limiting magnitudes scatter by \pm 0 m. 2 .

\subsection{Interactive screening}

The next step was the interactive screening of all detected objects. The aim of the selection is to provide the catalogue user with reference stars which are apparently undisturbed by neighbouring stars on the Schmidt plates. Selection was made by displaying $(25 \text { pixel })^{2}$ image frames centered on the preliminary object positions of 100 objects, so that some comparison of crowding conditions was possible. We excluded stars having close neighbours with separations of about $2^{\prime \prime}$ to $12^{\prime \prime}$. The efficiency of the screening against double stars varied somewhat with crowding conditions: in very crowded fields small perturbations were tolerated in order to keep a sufficient number of stars. Note that discarded stars might be undisturbed by their 
Table 1. Plate characteristics and number of objects. All plates were taken with the ESO Schmidt telescope (scale $67.52 / \mathrm{mm})$ with an exposure time of 60 minutes on IIa-O + GG 385

\begin{tabular}{|c|c|c|c|c|c|c|}
\hline \multirow[t]{2}{*}{ Field no. } & \multirow[t]{2}{*}{ Plate } & \multirow[t]{2}{*}{ Epoch } & \multicolumn{2}{|c|}{ Plate centre } & \multicolumn{2}{|c|}{ Number of objects } \\
\hline & & & $\alpha_{2000}[\mathrm{~h}]$ & $\delta_{2000}\left[^{\circ}\right]$ & detected & selected \\
\hline \multicolumn{7}{|c|}{ SMC } \\
\hline 28 & 9539 & 06.11.1991 & 0.0387 & -74.640 & 15504 & 13902 \\
\hline \multirow[t]{2}{*}{29} & 7745 & 12.12.1988 & 1.1211 & -74.699 & 16554 & 15494 \\
\hline & 7701 & 27.11.1988 & 1.1218 & -74.695 & 25172 & 18321 \\
\hline 50 & 9534 & 05.11 .1991 & 0.0399 & -69.700 & 12069 & 11135 \\
\hline 51 & 9544 & 07.11.1991 & 0.8964 & -69.583 & 15886 & 14281 \\
\hline \multirow[t]{2}{*}{52} & 8167 & 26.08 .1989 & 1.7602 & -69.836 & 15569 & 15425 \\
\hline & 7699 & 26.11 .1988 & 1.7491 & -69.561 & 12572 & 12115 \\
\hline \multicolumn{7}{|c|}{ LMC } \\
\hline \multirow[t]{2}{*}{33} & 7838 & 05.02 .1989 & 5.4870 & -74.755 & 20173 & 18564 \\
\hline & 7828 & 04.02.1989 & 5.4849 & -74.782 & 24951 & 21088 \\
\hline \multirow[t]{2}{*}{55} & 7844 & 07.02 .1989 & 4.3301 & -69.905 & 16649 & 15225 \\
\hline & 7839 & 06.02.1989 & 4.3301 & -69.909 & 15177 & 13558 \\
\hline \multirow[t]{2}{*}{56} & 7705 & 28.11.1988 & 5.1932 & -69.907 & 46652 & 34485 \\
\hline & 7702 & 27.11 .1988 & 5.1927 & -69.906 & 77671 & 54741 \\
\hline \multirow[t]{2}{*}{57} & 7797 & 08.01.1989 & 6.0568 & -69.949 & 34321 & 25536 \\
\hline & 7727 & 06.12 .1988 & 6.0587 & -69.956 & 27158 & 19625 \\
\hline \multirow[t]{2}{*}{84} & 7763 & 30.12 .1988 & 4.4115 & -64.923 & 15775 & 15356 \\
\hline & 7818 & 24.01.1989 & 4.4103 & -64.703 & 12321 & 11522 \\
\hline \multirow[t]{2}{*}{85} & 7819 & 28.01 .1989 & 5.1298 & -64.996 & 32582 & 28928 \\
\hline & 7820 & 29.01 .1989 & 5.1285 & -65.012 & 37037 & 26008 \\
\hline \multirow[t]{2}{*}{86} & 7824 & 30.01 .1989 & 5.8719 & -64.930 & 35937 & 26317 \\
\hline & 7734 & 08.12 .1988 & 5.8711 & -64.933 & 31390 & 21978 \\
\hline \multicolumn{2}{|c|}{ Sum: } & & & & 541120 & 433604 \\
\hline
\end{tabular}

neighbours on CCD frames due to a more favourable scale. What matters here, however, is reliable photographic reference positions. The screening is a major improvement relative to catalogues compiled by a fully automatic procedure like the GSC or the COSMOS/UKST Catalogue.

Galaxies, nebulae, star clusters, and artifacts were removed from the data base during this viewing. Therefore, the MACS does not contain any extended object. Bica \& Schmitt (1995) have compiled and unified different catalogues of extended objects in the SMC and the intercloud bridge and added their own discoveries. They give positions $\left(>5^{\prime \prime} \ldots 10^{\prime \prime}\right.$ accuracy), sizes, orientations, and cross-identifications for about 1200 clusters, associations, and nebulae (but no galaxies). These authors intend to publish a similar catalogue for the LMC in the near future.

Columns 6 and 7 of Table 1 give the numbers of detected and selected objects for each plate. A total of about 541000 objects were inspected, 434000 of which passed this procedure. The average selection rate was $80.1 \%$, with extremes of $99.1 \%$ and $70.0 \%$ depending on crowding conditions.

\section{Astrometry and catalogue construction}

In this chapter we describe how we compute and correct star positions for individual plates and how the positions of stars on different plates were combined for the final catalogue. The comparison between plates also gives an indication of the positional errors.

\subsection{Individual plates}

The computation of $(x, y)$-coordinates and internal magnitudes from small subframes containing the stars $(25 \times 25$ pixels for all stars and additionally $71 \times 71$ for the brighter ones) by one-dimensional Gauss fits to marginal distributions followed the standard procedure in Münster (Tucholke 1992). For typically $0.5 \%$ of the stars (up to $5 \%$ for one particularly crowded plate) the Gauss fit failed, and first-order moment positions were used instead. These positions were kept, however, since the final accuracy is given by the comparison to independent positions from other plates. 


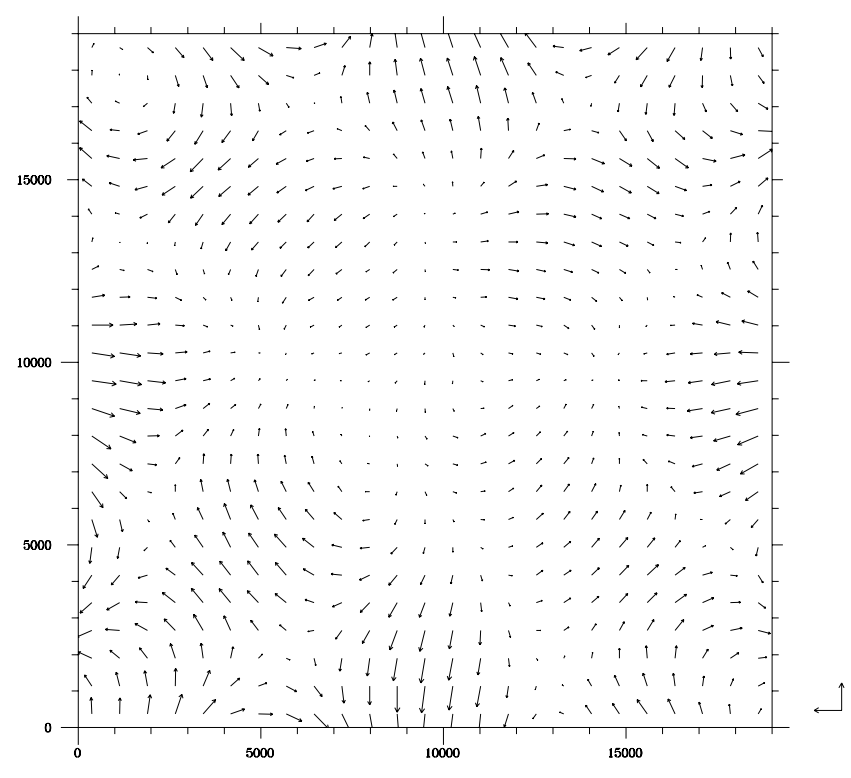

Fig. 3. Map of the distortions of ESO Schmidt plates relative to a second-order polynomial relating plate coordinates to the system of the PPM catalogue. The systematic pattern is probably caused by the bending of the plates during exposure. The coordinates were corrected for this effect before combining them into the MACS. The map was constructed by averaging the residuals of PPM stars from 21 plates in circles of 0.56 radius. The comparison arrows in the lower right corner have the length $5 \mu \mathrm{m}$ or 0. " $33.190001^{\prime \prime}$ pixels correspond to the plate size of $28.5 \mathrm{~cm}$ or 5.3 . North is up, East left

\subsubsection{Celestial coordinates}

Equatorial coordinates were derived by a classical singleplate adjustment. We used a second-order polynomial for the transformation of the $(x, y)$ to the reference system of the PPM South catalogue (Bastian et al. 1991). The Schmidt-telescope geometry was accounted for by the equidistant projection (Dick 1991). We used only PPM stars fainter than $m=9.0$, since bright stars on Schmidt plates show an asymmetrically located halo. Whether this is sufficient to prevent systematic differences between bright reference and faint target stars is investigated in Sect. 3.3.

After rejection of a small number of outliers, between 166 and 223 PPM stars per plate were used for the computation of the equatorial coordinates. The mean residuals from this transformation in $\alpha$ and $\delta$ were $0{ }^{\prime \prime} 22 \pm 0$ ". 02 (averaged over all reference stars on all plates).

\subsubsection{Correction for systematic errors}

Positions from Schmidt plates, obtained by transforming plate to celestial coordinates using low-order polynomi- als, frequently show systematic position-dependent residuals with respect to the reference catalogue. This effect has been described extensively by Taff et al. (1990b) (for earlier work see Dieckvoß \& de Vegt 1966 and Fresneau 1978 ) and has been demonstrated for various Schmidt telescopes (Taff et al. 1990a; Bucciarelli et al. 1992; Tucholke \& Schuecker 1992; Bienaymé 1993; Irwin 1994; Lopez Garcia et al. 1994; Abad 1995). The systematics are probably caused by the bending of the plates to the curved focal plane during exposure. Careful investigations have shown similar effects, presumably due to different reasons, for telescopes of the astrograph type as well (Abad 1993; Zacharias 1995; Smart et al. 1995).

A similar effect has also been detected in our case. Since all plates were exposed, developed and scanned in essentially the same way, we combined the residuals of PPM stars from all 21 plates into a two-dimensional array as a function of $x$ and $y$ (Fig. 3). This was done by averaging the residuals in circles of radius 2000 pixels (or 0 .56) for a regular $25 \times 25$ grid of test points in order to filter out the random errors. 3974 residuals were used with a minimum number of 50 residuals per bin.

Figure 3 shows clearly that systematic residuals of a complicated, but symmetric pattern are also present for the ESO Schmidt telescope. The mean systematic deviation as a function of position is $1.9 \mu \mathrm{m}\left(0^{\prime \prime} 13\right)$, while the maximum is $5.2 \mu \mathrm{m}\left(0^{\prime \prime} 35\right)$. $3.4 \%$ of the bins show residuals larger than $00^{\prime \prime} 25$. Various methods for the correction of such effects have been proposed, like the subplate method (Taff et al. 1990b; Smart et al. 1995), least-squares collocation (Bucciarelli et al. 1992), use of very high-order polynomials (Bienaymé 1993), mask method (Taff et al. 1990b), or moving numerical filter (Röser et al. 1995).

Since some of these methods need a very large number of reference stars, we used the simple mask method (also called réseau correction, Röser et al. 1995): the systematic pattern common to all plates is subtracted from the measured $(x, y)$-coordinates, and a new transformation from the corrected plate coordinates to celestial coordinates is computed. This method proved to be effective also in our case: The mean residuals decreased to 0.18 in both coordinates, and a residual map similar to Fig. 3 shows no traces of systematic errors larger than $1 \mu \mathrm{m}$. Although individual plates do not strictly adhere to this systematic pattern, an individual correction for each plate is not feasible, since the number of PPM stars per plate is too low to allow sufficient spatial resolution.

\subsection{Plates of the same field}

For nine of our fields we used two plates each. For combining the lists of positions and internal magnitudes, we used a tolerance radius of $5^{\prime \prime}$. Since stars disturbed by close neighbours in the separation range $2^{\prime \prime}$ to $11^{\prime \prime}$ have been removed, and since epoch differences are negligible, the identification is unambiguous. 
The mean differences in right ascension and declination turned out to be insignificant compared to the rms differences, which are $00^{\prime \prime} 15$ in $\alpha$ and $0^{\prime \prime} 14$ in $\delta$, averaged over the fields. Extrema are $0{ }^{\prime \prime} 11$ and $0{ }^{\prime \prime} 20$. The rms scatter of internal magnitudes about the mean difference, which is caused by slightly different limiting magnitudes, ranges from $0 .{ }^{\mathrm{m}} 11$ to 0.20 , with a mean of $0 .{ }^{\mathrm{m}} 15$.

\subsection{Field-to-field overlaps}

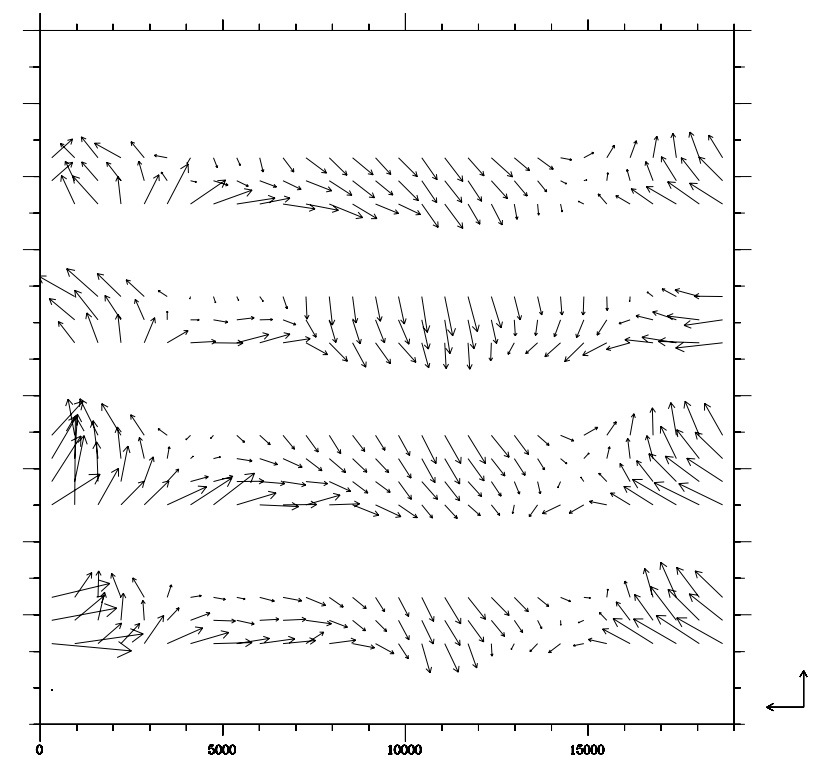

Fig. 4. Differences of positions between plates overlapping in declination, averaged over six overlap regions and in circles of radius 1000 pixels. From top to bottom, the four plots correspond to approximate $B$ magnitude ranges 10 to $13,13.5$ to $14.0,14.5$ to 15 , and 16.0 to 16.5 . They are shifted by an arbitrary amount in $y$ for clarity. The compared positions are already corrected for the systematic pattern seen in Fig. 3, which is common to all plates. The differences shown here are probably caused by the fact that the reference stars used for the astrometric reduction of the plates have a magnitude range very different from that of typical MACS stars (see text). The comparison arrows at the lower right of the plot have the length $5 \mu \mathrm{m}$ or $0 . ' 33$. Tick marks on the axes are in steps of 1000 pixels

Neighbouring fields overlap slightly (see Figs. 1 and 2). The data for stars in the overlap regions were averaged during the assembly of the final catalogues. Again, an identification radius of $5^{\prime \prime}$ was used. The overlap regions provide an extreme test for the positional accuracy, since positions near the plate edges are compared, where photographic astrometry usually has its least accuracy.

Figure 4 shows the mean positional differences between neighbouring plates from six overlaps in declination. The differences have been averaged in circles of radius 1000 pixels in order to suppress the random errors. Plots for four ranges of magnitude, shifted in $y$ for clarity, are given. Each of the plots is based on 500 to 1000 stars.

Although all celestial coordinates have already been corrected for the systematic pattern common to all plates (Fig. 3), the plots show that positions from overlapping plates still differ systematically. Mean systematic differences are $3.5 \mu \mathrm{m}$ or $00^{\prime \prime} 24$. There seems to be a tendency for increasing systematic differences with fainter magnitudes. The probable reason for this effect is the magnitude difference between reference stars (9 to 10.5) and MACS stars (typically 15 to 16 ). The same centering algorithm was used for all stars, while the reference stars, even those with magnitudes fainter than 9.0 used here, apparently have an asymmetric density distribution, an effect increasing with distance from the plate centre $r$. Thus we expect a systematic positional displacement between faint and bright stars, increasing with $r$ and probably also with magnitude. The systematic pattern for right ascension overlaps looks similar. Qualitatively similar results have been found by Drinkwater et al. (1995) and Morrison et al. (1996).

We are left with position-dependent systematics which cannot be removed at present, since no faint and dense reference catalogue is available. A magnitude limit for PPM stars significantly fainter than 9.0 would leave us with a too small number of reference stars. This systematic effect limits the positional accuracy of the catalogue, at least near the field boundaries. We estimate a conservative upper limit to the non-random positional errors of 0 "' 33 . For a typical MACS star the positions should be better, since a) the final positions result from averaging in overlap regions, which should reduce the error in most cases, b) the non-overlapping plate areas are expected to be less affected by systematic errors of this kind (only $12 \%$ of the catalogue stars are located in overlap regions).

Although the positional differences in overlap regions contain a dominant contribution from position-dependent systematics, we give the rms differences over the complete strips. The mean over 15 overlaps is 0 '! 23 in $\alpha$ as well as in $\delta$ with extremes at 0.18 and $0 . \prime 31$.

The internal magnitude zero point differs between plates by 0.2 on the average. The rms scatter about the mean difference is $0{ }^{\mathrm{m}} 14$ with extremes at $0{ }^{\mathrm{m}} 09$ and $0{ }^{\mathrm{m}} 19$. After correcting the internal magnitudes of the different fields to a common zero point for each region (LMC and $\mathrm{SMC}$ ), the values from different plates were averaged, and means and standard deviations computed.

\subsection{Comparison with Périé et al.}

Périé et al. (1991) presented a catalogue of 926 stars towards the LMC and the SMC with $V \leq 11$. They used 27 ESO Schmidt plates and the Perth70, which is on the FK4 system, as reference catalogue. For comparison, their positions were brought to the FK5/J2000.0 system, on which the PPM is based. We excluded bright stars with $V \leq 7$. 
After purging a few outliers, the mean differences in the sense (MACS-Périé) are:

$$
\begin{aligned}
& \text { LMC : } \quad \Delta \alpha \cos \delta=-0 \text { ". } 13 \pm 0 . .33 \quad(N=613) \\
& \Delta \delta=+0.22 \pm 0.40 \\
& \mathrm{SMC}: \quad \Delta \alpha \cos \delta=+0 . \prime 04 \pm 0.43 \quad(N=171) \\
& \Delta \delta=+0^{\prime \prime} \cdot 04 \pm 0.35
\end{aligned}
$$

The scatter of the differences is fully explained by the internal errors of both catalogues, which are 0 ". 43,0 ". 44 for Périé et al. and 0 "' 23 for our catalogue, leading to an expected scatter of 0.449 and $0 ., 50$ in $\alpha$ and $\delta$. In the case of the LMC the offset is significant compared to its error, which results from dividing the given rms scatter by the square root of the number of stars used. A magnitude dependent systematic error in (at least) one of both catalogues could be a possible reason. Differences of this size have also been found in other comparisons of astrometric catalogues.

\section{Contents of the catalogue}

The Magellanic Catalogue of Stars (MACS) consists of two parts, one for the LMC region with 175779 stars and one for the SMC with 67782 entries. Each star has one entry only. The catalogues are ordered by increasing right ascension. The surface density of stars in low-density regions is 0.15 stars $/ \square^{\prime}$. This is just sufficient to yield for a present day CCD image covering about $25 \square^{\prime}$ a minimum number of 4 reference stars.

In Table 2 we give, as an example, some lines from the MACS for the regions around the globular cluster NGC 330 (SMC) and near SN 1987a (LMC). The columns are explained below.

\subsection{Nomenclature}

Column 1 is the star name following the proposal of de Boer (1993), giving its truncated position and a running number within an $(\alpha, \delta)$-box of $1^{\mathrm{m}} \times 6^{\prime}$. For example, a star with $(\alpha, \delta)=\left(5^{\mathrm{h}} 47^{\mathrm{m}} 02^{\mathrm{m}} 4,-67^{\circ} 12^{\prime} 02^{\prime \prime}\right)$ would have the designation MACS J0547-672\#001, if it is the first star in its box. The positional coding uses in right ascension the hhmm format and in declination decimal degrees (truncated!), thus defining (in the southern sky) the NW corner of the box. In this first edition of the MACS the sequential number runs along $\alpha$ in the box; this is, however, not a requirement. Further stars inside the box can be added to the catalogue without upsetting the numbering system. This format is in agreement with the Rules and Regulations for Nomenclature as defined by IAU Commission 5 (see the A\&A issues with the Annual Index). Currently, the largest number of stars within a box is 84 and 40 for the LMC and the SMC, respectively.

\subsection{Description of columns}

Columns 2 and 3 list right ascension and declination for the equinox J2000.0 (FK5/PPM system). The epoch is the average of the plate epochs, which is around 1989.0. For some SMC fields $(28,50,51)$ the epoch is 1991.8. This difference matters only for stars of very high proper motion. Column 4 gives the number of positions, from which $\alpha, \delta$, and $m$ were computed. $N$ may reach 8 for a fourfold corner overlap; on the average one MACS position is derived from measurements on 1.8 plates. Column 5 lists the internal magnitude, whose zero points for the two catalogue parts are arbitrary; they should be useful at least for ranking the stars by blue magnitude. For stars where the magnitude measurement failed, a dummy value of 99.0 is given.

We refrained from providing internal errors of positions and magnitudes, since they are likely not of interest for the general user, thus saving space. Instead, we give flags for stars whose internal errors are exceptionally large. Column 6 is the bad position flag, where 0 is ok, and 1 signals an internal error $>0.5$. $2394=0.99 \%$ of stars carry this flag. Similarly, in column 7 a flag $=1$ means that the internal magnitude is larger than a magnitude-dependent limit chosen to select only exceptionally high values. 1248 $=0.51 \%$ of the stars carry that flag. Flag $=1$ may also point to a large-amplitude photometric variation.

Finally, column 8 is 1 , if the star has an entry in the data base of astrophysical information on relatively bright LMC (member and foreground) stars, which is currently being compiled at the Astrophysikalisches Institut Bochum (Gochermann et al. 1995). It contains data for about 10000 stars with $V \leq 12$. The cross-identification with this data base is still pending, but should be accomplished in the near future.

\subsection{Access}

The MACS in its two parts is available from the Centre des Données Strasbourg (CDS) via ftp cdsarc.u-strasbg.fr in the directory I/221. The upto-date version may also be downloaded via anonymous ftp from the Ultrix Cluster of the Astronomical Institutes of Bonn University by ftp ftp.astro.uni-bonn.de in the directory pub/macs. At present, no dedicated search software is available to outside users.

\section{Discussion}

The MACS has been used successfully as astrometrical reference for CCD frames in selected regions of the LMC (Will et al. 1995). The catalogue also served as a source of target coordinates for IUE observations of bright stars near LMC clusters. In these crowded regions a difficulty in practical use became apparent: Many stars, among them bright ones, are missing from the MACS, since they were 
Table 2. Some lines from the preliminary MACS for stars in the fields of NGC 330 (SMC) and SN 1987a (LMC). The name of the object consists of the acronym MACS (for Magellanic Catalogue of Stars, but one can also read it as Magellanic Cloud Star), the letter J denoting the equinox J2000.0 to which the position refers, a position code for the upper right (NW) corner of the box with size $1^{\mathrm{m}} \times 6^{\prime}$, and the \#nnn giving the running number of the star inside that box. For further explanation of the columns see text

\begin{tabular}{|c|c|c|c|c|c|c|c|}
\hline \multirow[t]{2}{*}{ Name } & \multirow[t]{2}{*}{$\alpha_{2000}$} & \multirow[t]{2}{*}{$\delta_{2000}$} & \multirow[t]{2}{*}{$N$} & \multirow{2}{*}{$\begin{array}{l}\text { Int. } \\
\text { Mag. }\end{array}$} & \multicolumn{3}{|c|}{ Flags } \\
\hline & & & & & Pos & Mag & Bochum \\
\hline$\vdots$ & $\vdots$ & $\vdots$ & & & & & \\
\hline MACS J0056-724\#003 & 05603.321 & -722907.80 & 1 & 16.56 & 0 & 0 & 0 \\
\hline MACS J0056-724\#005 & 05606.837 & -722835.25 & 1 & 17.81 & 0 & 0 & 0 \\
\hline MACS J0056-724\#006 & 05607.449 & -722928.38 & 2 & 18.19 & 0 & 0 & 0 \\
\hline MACS J0056-725\#005 & 05613.847 & -723000.32 & 2 & 17.49 & 0 & 0 & 0 \\
\hline MACS J0056-724\#010 & 05624.076 & -722914.48 & 2 & 17.04 & 0 & 0 & 0 \\
\hline MACS J0535-692\#022 & 53530.401 & $-69 \quad 16 \quad 18.13$ & 1 & 19.33 & 0 & 0 & 0 \\
\hline MACS J0535-692\#024 & 53532.625 & -691548.53 & 4 & 19.14 & 0 & 0 & 0 \\
\hline MACS J0535-692\#025 & 53534.493 & -691713.58 & 1 & 99.00 & 0 & 1 & 0 \\
\hline MACS J0535-693\#024 & 53534.828 & -691919.53 & 1 & 19.98 & 0 & 0 & 0 \\
\hline MACS J0535-692\#027 & 53536.136 & -691608.96 & 4 & 17.98 & 0 & 0 & 0 \\
\hline$\vdots$ & $\vdots$ & $\vdots$ & & & & & \\
\hline
\end{tabular}

rejected due to crowding. This makes the comparison with object lists from other sources difficult. Once the identification with a few MACS stars is done, coordinates of the remaining stars in the field of interest can be obtained by relating relative positions from pre-existing CCD frames to those of the catalogue stars. The problem of identification could be alleviated by providing zoomed-down versions of the original scans, with MACS stars marked.

An important cross-identification of the MACS will be with GSC 1.0 and its successor versions, which will give further hints on the astrometric accuracy of both catalogues. On the astrophysical side, cross-identifications with catalogues of variables, high-proper motion stars, or MK spectral classifications will be of interest. The relation to the Bochum data base of bright LMC stars has already been mentioned in the previous section.

In the current version, the MACS lacks information on colour and proper motion. Crude proper motions with an accuracy useful for separating foreground stars can be obtained by scanning the respective Quick Blue Survey plates, which is in progress. Colour information will require the use of different plate material.

Acknowledgements. We are indebted to the staff of the ESO Schmidt telescope for taking the photographic plates used in this work. Comments by S. Röser improved the astrometric section of this article. Discussions with P. Dubois, M.C. Lortet, and F. Spite were helpful in defining the final nomenclature. F. Ochsenbein and S. Jeffery helped to debug the on-line version of the catalogue.

\section{References}

Abad C., 1993, A\&AS 98, 1

Abad C., 1995, A\&AS 111, 369

Bastian U., Röser S., Nesterov V.V., et al., 1991, A\&AS 87, 159

Bica E.L.D., Schmitt H.R., 1995, ApJS 101, 41

Bienaymé O., 1993, A\&A 278, 301

Bucciarelli B., Daou D., Lattanzi M.G., Taff L.G., 1992, AJ 103, 1689

de Boer K.S., 1993, in "Recent Advances in Magellanic Cloud Research". In: Baschek B., Klare G., Lequeux J. (eds.). Heidelberg: Springer, p. 389

Dick W.R., 1991, Astron. Nachr. 312, 113

Dieckvoß W., de Vegt C., 1966, Abhandlungen Hamburger Sternwarte 8, 82

Drinkwater M.J., Barnes D.G., Ellison S.L., 1995, Publ. Astron. Soc. Austr. 12, 248

Fresneau A., 1978, AJ 83, 406

Gochermann J., Grothues H.-G., Oestreicher M.O., SchmidtKaler Th., 1995, Astron. Ges. Abstr. Ser. 11, 209

Horstmann H., 1992, Ph. D. Thesis, Astron. Inst. Univ. Münster (in German)

Irwin M., 1994, Working Group Wide-Field Imaging Newslett. 5,25

Lasker B.M., Sturch C.R., McLean B.J., et al., 1990, AJ 99, 2019 
Lopez Garcia A., Martinez Gonzalez J.M., Ortiz Gil A., Yagudin L.I., 1994, Working Group Wide-Field Imaging Newslett. 6, 11

Morrison J.E., Röser S., Lasker B.M., Smart R.L., Taff L.G., 1996, AJ 111, 1405

Périé J., Prévot L., Rousseau M., Peyrin Y., Robin A., 1991, A\&AS 90, 1

Röser S., Schilbach E., Hirte S., 1995, in ESA SP-379 "Future possibilities for astrometry in space". In: Perryman M.A.C., van Leeuwen F. (eds.), Noordwijk: ESA Scientific Publications Division, p. 143

Smart R.L., Taff L.G., Morrison J.E., 1995, AJ 110, 2469

Taff L.G., Lattanzi M.G., Bucciarelli B., et al., 1990a, ApJ 353, $\mathrm{L} 45$
Taff L.G., Lattanzi M.G., Bucciarelli B., 1990b, ApJ 358, 359

Tucholke H.-J., 1992, A\&AS 93, 293

Tucholke H.-J., Hiesgen M., 1991, in IAU Symposium 148 "The Magellanic Clouds". In: Haynes R.F. \& Milne D.K. (eds.). Dordrecht: Kluwer, p. 491

Tucholke H.-J., Schuecker P., 1992, PASP 104, 704

West R.M., Schuster H.-E., 1982, A\&AS 49, 577

Will J.-M., Bomans D.J., Tucholke H.-J., et al., 1995, A\&AS 112,367

Yentis D.J., Cruddace R.G., Gursky H., et al., 1992, in "Digitized optical sky surveys". In: MacGillivray H.T. \& Thomson E.B. (eds.). Dordrecht: Kluwer, p. 67

Zacharias N., 1995, AJ 109, 1880 